\title{
Role of Ceramides in Nonalcoholic Fatty Liver Disease
}

\author{
Mangesh Pagadala ${ }^{1}$, Takhar Kasumov ${ }^{1}$, Arthur J. McCullough ${ }^{1}$, Nizar N. Zein ${ }^{1}$, and John P. \\ Kirwan $^{1,2,3,4}$ \\ ${ }^{1}$ Department of Gastroenterology/Hepatology, Cleveland Clinic, Cleveland, OH, 44195 \\ ${ }^{2}$ Department of Pathobiology, Lerner Research Institute, Cleveland Clinic, Cleveland, $\mathrm{OH}, 44195$ \\ ${ }^{3}$ Department of Nutrition, Case Western Reserve University, School of Medicine, Cleveland, $\mathrm{OH}$, \\ 44106 \\ ${ }^{4}$ Metabolic Translational Research Center, Endocrinology and Metabolism Institute, Cleveland \\ Clinic, $\mathrm{OH}, 44195$
}

\begin{abstract}
Nonalcoholic fatty liver disease (NAFLD) is a chronic disease with a histological spectrum ranging from steatosis alone, to nonalcoholic steatohepatitis (NASH). The latter is associated with an increased risk for progression to cirrhosis. Ceramides are a lipid species that exert biological effects through cellular proliferation, differentiation and cell death, and interact with several pathways involved in insulin resistance, oxidative stress, inflammation, and apoptosis, all of which are linked to NAFLD. Herein, we propose a mechanism through which ceramides contribute to the development of NAFLD and progression to NASH, due in part to second messenger effects via TNF- $a$. A better understanding of the role of ceramides in steatohepatitis has both diagnostic and therapeutic implications for the treatment of fatty liver disease.
\end{abstract}

\section{Keywords}

Insulin resistance; obesity; sphingolipids; apoptosis; oxidative stress

\section{Ceramides and the pathogenesis of nonalcoholic fatty liver disease}

NAFLD is a chronic liver disease with a histological spectrum ranging from steatosis (accumulation of hepatic fat) alone, to nonalcoholic steatohepatitis (NASH), both of which are consistent with hepatocyte injury, necroinflammation and fibrosis. The latter is associated with an increased risk for progression to cirrhosis [1]. NAFLD has been reported in $17-33 \%$ of the general population making it one of the most common chronic liver diseases in the US, while estimates for NASH suggest it is present in 5-17\% of the population [1]. The deposition of hepatic lipids, especially triacylglycerol, defines the development of hepatic steatosis and NAFLD [2]. However, there is emerging evidence that other lipid moieties such as ceramides also play an important role in NAFLD and possibly progression to NASH.

\footnotetext{
(C) 2012 Elsevier Ltd. All rights reserved.

Corresponding Author: John P. Kirwan, Ph.D., Department of Pathobiology, Lerner Research Institute, Cleveland Clinic, 9500 Euclid Avenue / NE-40, Cleveland, OH 44195, kirwanj@ ccf.org, Telephone: 216-444-3412, Fax: 216-636-1493.

Publisher's Disclaimer: This is a PDF file of an unedited manuscript that has been accepted for publication. As a service to our customers we are providing this early version of the manuscript. The manuscript will undergo copyediting, typesetting, and review of the resulting proof before it is published in its final citable form. Please note that during the production process errors may be discovered which could affect the content, and all legal disclaimers that apply to the journal pertain.
} 
Ceramides are members of the sphingolipid family of lipids and are integral to the structure of the lipid bilayer that makes up cell membranes [3]. However, ceramides also have cellsignaling properties, and their accumulation in the liver especially during periods of increased hepatic influx of free fatty acids (FFA) may contribute to insulin resistance [4]. While patients with NAFLD share many of the features of metabolic syndrome such as insulin resistance, obesity, hypertension and dyslipidemia, it is thought that insulin resistance and oxidative stress are primary mediators in the progression to NASH [5]. Since ceramides inhibit insulin signaling, and induce oxidative stress and inflammation, they too may play a role in the development of NAFLD and progression to NASH [6, 7]. However, knowledge of the role of ceramides in the pathogenesis of NASH is still evolving.

In this article, we draw on data published in the literature that links ceramides with risk factors for NASH, and suggest a possible role for ceramides in the pathogenesis of NASH either directly, or as an important second messenger. In addition, we also review the possible role of ceramides in hepatic apoptosis, which is a key feature of NASH and serves to promote hepatic inflammation and fibrosis. There is some consensus that the pathogenesis of NAFLD is linked to a "two-hit hypothesis", in which the "first hit" is associated with the development of central obesity and then insulin resistance, both of which increase the vulnerability of the liver to various "second hits", notably oxidative stress and the production of inflammatory cytokines $[1,5]$. Patients with NASH also have hepatic mitochondrial dysfunction, as characterized by impaired oxidative phosphorylation [8]. Oxidative stress can lead to mitochondrial dysfunction and contribute to hepatic apoptosis, which in turn exacerbates inflammation and in many cases leads to liver fibrosis [8,9]. It is important to emphasize that while steatosis itself usually has a relatively benign course, it can sensitize the liver to the more damaging effects of insulin resistance, cytokines, or oxidative stress, and lead to the development of NASH [10]. Interestingly ceramides have been linked to the different entities that cause the "second hit", and here, we propose a hypothesis around the two hit model suggesting a role for ceramides in the development and progression of NAFLD.

\section{Lipotoxicity and the production of ceramides in the liver}

Ceramides are generated through three different pathways; de novo synthesis, a sphingomyelinase (SMase) pathway, and a salvage pathway (Fig. 1). The de novo pathway can be induced by metabolic loading with serine, oxidative stress, and oxidized LDL [11]. Ceramide synthesis from palmitoyl-CoA and serine involves four sequential reactions catalyzed by serine palmitoyl-CoA transferase (SPT), 3-oxosphinganine reductase, sphingosine $\mathrm{N}$-acyltransferase (ceramide synthase) and dihydroceramide desaturase. SPT, the rate-limiting enzyme of de novo synthesis, has high substrate specificity for palmitoylCoA. Thus, a diet rich in saturated fat can stimulate ceramide synthesis [12]. Cellular redox status regulates de novo ceramide synthesis through inhibition of dihydroceramide desaturase by the antioxidant, glutathione (GSH) [11]. Therefore, infection (lipopolysacharide), induction of inflammatory cytokines (TNF- $a$ and IL-1), and oxidative stress can all increase mRNA expression and activity of SPT in macrophages, and in turn activation of ceramide synthesis [13]. Ceramide itself can also regulate pro-inflammatory gene expression through activation of the transcription factor known as nuclear factor kappa-light-chain-enhancer of activated B cells (NF- $\mathrm{B}$ ); this promotes a powerful positive feedback mechanism whereby inflammation is sustained and amplified [4]. This is in agreement with recent observations that ceramides are required for TLR4 dependent insulin resistance [14].

As a second messenger in the SMase transmembrane signaling pathway, ceramide is also generated by the neutral sphingomyelinase (N-SMase)-catalyzed hydrolysis of 
sphingomyelin in cell membranes. In addition to activation of de novo ceramide synthesis, inflammation up-regulates activity of N-SMase $[15,16]$. GSH can act as a negative regulator of N-SMase and reduced GSH leads to N-SMase activation [17]. Ceramide may also be recycled by the ceramide synthase catalyzed reacylation of sphingosine generated from catabolism of complex sphingolipids (salvage pathway). Fumanisin B1, an inhibitor of (dihydro)ceramide synthase, inhibits both ceramide biosynthesis and the salvage pathways.

While ceramide synthesis occurs in virtually all organs in the body, there are some important data from cell lines, animal models, and human studies suggesting that liver is a key site for ceramide production [18-20]. Treatment of cultured rat hepatocytes with proinflammatory cytokines like interleukin-1 $\beta$ (IL-1 $\beta$ ) causes a rapid turnover of both sphingomyelin as well as intracellular ceramide content [21]. In mouse models, induction of hepatic necrosis by carbon tetrachloride results in significant increases in both plasma and hepatic ceramide concentrations [22]. Furthermore, generation of acute phase response (APR) proteins in mice by stimulation with lipopolysaccharides (LPS) leads to an increase in hepatic ceramide production [18]. Similar results were noted in human studies where an increase in plasma ceramide content is seen to correlate well with an increase in liver SPT and serum acid sphingomyelinase activity [23]. In the same study up to $80 \%$ of plasma ceramides were associated with VLDL and LDL, both of which originate in the liver.

The key pathological feature in the development of hepatic steatosis is up-regulation of peripheral adipose tissue lipolysis leading to an increased influx of FFAs into the liver [24, 25]. These FFAs can be converted into a number of different lipid species including triglycerides, diacylglycerol and sphingolipids, or undergo oxidation in mitochondria, microsomes, or peroxisomes [2]. Ultimately, triglycerides are exported out of the liver as VLDL. However, upon increased hepatic influx of FFAs, as noted in insulin resistance, the rate of increase in FFA flux exceeds secretion as VLDL, or fat oxidation, resulting in the progressive increase in hepatic lipids including triglycerides and ceramides [25]. It is thought that this ectopic lipid deposition can lead to cell dysfunction and cell death, a phenomenon described as lipotoxicity [26]. In patients with NAFLD, the hepatic concentration of saturated fatty acids like palmitic acid and mono-unsaturated fatty acids like oleic acid are significantly increased [25, 27]. When H4IIE rat liver hepatoma cells are exposed to varying concentrations of saturated fatty acids (palmitate or stearate) there is a significant accumulation of cermides in the cells [28]. Recently, Chocian et al. demonstrated that when rats were fed a high fat diet enriched in saturated fatty acids, the liver and hepatic nuclei accumulated significant amounts of ceramide and sphingomyelin [29]. An increase in the enzymes involved in ceramide metabolism including SMase and sphingomyelin synthase in nuclear membranes isolated from rat liver may help to explain the increased synthesis of ceramides in hepatic nuclei [30]. Obese mice with hepatic steatosis also have increased hepatic concentrations of ceramides [31]. Blocking de novo ceramide synthesis using myriocin reduced macrovesicular fat accumulation, hepatic triglyceride content and expression of SOC-3, a cytokine mediated gene that is also upregulated in NAFLD [32]. The type of fatty acid also effects ceramide synthesis, as a 6-hour infusion of lard oil (saturated fatty acids), but not soy-based infusate (predominantly unsaturated fatty acids), resulted in a $60 \%$ increase in hepatic ceramide content in rats, compared to controls [33]. The generation of ceramides in the liver may also be mediated by cytokines like TNF- $a$, and various associated death ligands. The FAS ligand (CD95L) is a type-II transmembrane protein that belongs to the TNF-a receptor family. It is involved in caspase-mediated apoptosis and can activate inflammatory pathways in various cell lines [34]. Importantly, when adipose tissue specific FAS knockout mice are fed a high fat diet there is decreased expression of CD36, an important fatty acid transport protein, as well as decreased liver lipid content including total ceramide, further suggesting a possible link between hepatic FFA entry and ceramide production [35]. In the context of the above observation it is unclear if hepatic steatosis is a 
direct result of ceramide accumulation in the liver, or rather due to increased ceramide mediated insulin resistance in adipose tissue that leads to increased plasma FFA and lipotoxicity, features that are also noted in NASH.

\section{Effects of ceramide on insulin sensitivity}

Individual risk factors that contribute to metabolic syndrome, like insulin resistance and central obesity, are known to be good predictors of NASH progression [36]. Peripheral insulin resistance promotes lipolysis and increased FFA availability to the liver, thus facilitating hepatic triglyceride synthesis [2]. At the cellular level, insulin resistance is caused by impaired signaling in the canonical insulin signaling cascade, which in turn leads to impaired glucose uptake into skeletal muscle and suppression of glucose output from the liver, i.e., "hepatic insulin resistance", a phenomenon directly associated with NAFLD [37]. Ceramides may contribute to whole body insulin resistance by attenuating insulin signaling in skeletal muscle and the liver [33]. Ceramides have been shown to impair insulin sensitivity in skeletal muscle cells by reducing GLUT4 translocation and glucose uptake [38]. Evidence to support ceramide regulation of insulin signaling and insulin sensitivity in skeletal muscle and liver include pharmacological inhibition of sphingolipids, which leads to increased phosphorylation of the insulin receptor, Akt and mTOR [18]. Further, studies using cultured skeletal muscle cells and animal models show increased activation of protein phosphatase 2A (PP2A), inhibition of Akt activity and subsequent sequestration from the cytoplasm to the plasma membrane $[4,18,39]$. Although the primary mechanism by which ceramides induce hepatic insulin resistance is not completely understood, exposure of cultured hepatocytes to short chain ceramides blunts insulin mediated activation of Akt, as well as glucokinase, an important enzyme that regulates insulin mediated glucose uptake and subsequent conversion to glycogen [40]. Inhibition of ceramide biosynthesis using myriocin, in diet induced obese mice, led to enhanced hepatic insulin signaling and increased basal and insulin stimulated Akt phosphorylation in the liver [32]. Similarly, Holland et al. found that dexamethasone and lipid infusion (saturated fatty acids) promotes ceramide accumulation in rat liver. Further, treatment with dexamethasone led to impaired Akt signaling both in liver and muscle without affecting PI 3-kinase, and these effects were reversed by myriocin. It has also been shown that heterozygous dihydroceramide desaturase (an enzyme required to convert inactive dihydroceramide into active ceramide) deficient mice are protected against steroid-induced hepatic ceramide accumulation, and these animals also have better glucose control compared to wild type controls [18, 33]. Collectively, these studies support the case for ceramide mediated insulin resistance in tissues including the liver and suggests that the effect is being induced through Akt inactivation. Additional support for a link between ceramide and hepatic insulin resistance may be gleaned from data showing that ceramides participate in insulin signaling upstream of Akt by up-regulating inhibitory serine kinases including, inhibitor of $\kappa \mathrm{B}$-kinase- $\beta$ (I $\mathrm{kBk}$ ) and cJun $\mathrm{N}$-terminal kinase (JNK), known inhibitors of the insulin signaling cascade [41, 42]. While there is good evidence that ceramides induce skeletal muscle insulin resistance through Akt, there is less data to support this mechanism in the liver. We conjecture that insulin resistance in the liver is regulated through Akt, and this occurs directly through either endogenous ceramides, or indirectly through circulating ceramides.

\section{Ceramides and cytokine-mediated hepatic inflammation}

Pro-inflammatory cytokines such as TNF- $a$ can initiate ceramide synthesis by binding to TNFR1 and activating ASMase and possibly N-SMase [7]. Interaction between TNF- $\alpha$ and ceramides, besides causing insulin resistance, also increases mitochondrial generation of ROS, thus promoting apoptosis and a further recruitment of inflammatory cells to the liver, leading to a worsening of hepatic inflammation [43]. An increase in circulating levels of 
another pro-inflammatory cytokine IL-6 is also associated with severity of NASH and correlates well with plasma ceramides [44, 45]. Further, the pro-inflammatory cytokine IL-1 is increased in NASH, and it also induces ceramide production [18].

Adiponectin is an anti-inflammatory cytokine that can improve insulin sensitivity through inhibition of hepatic gluconeogenesis, suppression of lipogenesis, and enhanced oxidation of FFAs within the mitochondria, thus decreasing hepatic lipid accumulation [46, 47]. Adiponectin also mitigates TNF- $a$ and promotes IL-10 release from Kupffer cells [47, 48]. Interestingly, in a recent study in diet-induced obese mice, total hepatic ceramide levels were significantly reduced by intravenous adiponectin administration [49]. Adiponectin, acting through adipoR 1 and adipoR2 receptors, decreased ceramides by up-regulating ceramidase, the enzyme that converts ceramide to sphingosine-1-phosphate. The resulting improvement in hepatic insulin sensitivity, highlights the mechanistic link between inflammation and insulin resistance, and demonstrates how ceramides can be targeted to improve metabolic function.

When examining the role of ceramide in the development of steatohepatitis, it is prudent to consider that while chronic hepatic inflammation and increased TNF-a may initiate ceramide production in hepatocytes, there is also potential for increased ceramide to regulate cytokine secretion by its own feedback mechanism. Therefore, with inflammation, there is an interaction between TNF- $a$ and ceramide that is part of a vicious cycle that may synergistically potentiate the damaging metabolic effects caused by either compound on its own. Taken together there is evidence that ceramide may play an important role in steatohepatitis via its interaction with cytokines, however this mechanism needs further validation in humans.

\section{Role of ceramides in mitochondrial ROS production}

Mitochondria are a major site of oxygen consumption, and ROS generation in patients with NAFLD [50]. In NASH, structural abnormalities in mitochondria are thought to be an adaptive response to this oxidative stress [51]. Respiratory chain dysfunction with subsequent formation of oxygen radicals is a major source of ROS [51, 52]. In cell systems, short chain ceramides are toxic to isolated mitochondria because of increased permeability of mitochondrial membranes, the formation of ceramide channels and increased cytochrome $\mathrm{C}$ release [53]. Further, GSH depletion by TNF- $\alpha$ in the matrix may sensitize mitochondrial hepatocytes to ceramide-mediated damage [54, 55]. It is thought that ASMase plays an important role in generating ROS and this production may be regulated by TNF-a [4, 7]. In hepatocytes from ASMase - $/-$ mice, TNF- $a$ did not generate an increase in ROS production despite the absence of the antioxidant GSH [55]. These data reinforce the link between ceramides and TNF- $a$ in ASMase regulation of mitochondrial dysfunction. Furthermore, ceramides can directly regulate mitochondrial respiration by inhibiting the ubiquinone pool of complex III as well as nicotinamide adenine dinucleotide phosphate oxidase (NADPH oxidase) [18]. While accumulation of ceramides within the cells may target mitochondria, there is also evidence that enzymes including ceramide synthase are present on the outer membrane of mitochondria isolated from hepatocytes [56, 57]. Therefore, we speculate that the action of mitochondrial mediated ROS production through ceramide occurs as a result of accumulation of sphingolipids in the cytoplasm, and in part may be due to de novo ceramide production within the mitochondria. The mechanism of TNF- $a$ induced mitochondrial damage may also require ganglioside GD3, which is a ceramide-derived sphingolipid [58].

\section{Ceramides and hepatic apoptosis}

In NAFLD, there is mitochondrial instability and this is thought to trigger the apoptotic pathway setting up a cascade of events resulting in inflammation, repair, and hepatocyte 
regeneration [59]. Apoptosis has been linked to fibrogenesis in human and rat models, secondary to the engulfment of the apoptotic cell (balloon hepatocyte) by hepatic stellate cells [59-61]. Additionally, apoptosis is associated with histological severity in NASH, and the caspase pathway has been implicated as a primary mechanism of hepatocyte apoptosis [62]. In various cell lines, different stimuli like ionizing radiation, ultraviolet light, chemotherapeutic agents, oxidative stress, TNF-a, and FAS ligands, are associated in cell death pathways that use ceramides as a second death messenger [3, 63]. Moreover, in humans with NASH, up-regulation of death receptors like FAS and TNFR1 are involved in hepatocyte apopotosis [59, 64]. Cell death mediated by TNF-a employs ceramide as an important second messenger, and this has been demonstrated in HeLa, fibroblast, and lymphoblast cell lines. In these models, TNF-a binds to TNFR1 and activates lysosomal ASMase, thus promoting ceramide formation, which in turn activates 52KD procathepsin D, converting it to the more active $32 \mathrm{KD}$ cathepsin. The entry of cathepsin $\mathrm{D}$ into the cytosol leads to an increased permeabilization of mitochondria, releasing cytochrome $\mathrm{C}$ and thereby triggering caspase-induced apoptosis [65]. While it is clear that ceramides induce apoptosis in non-hepatic cells through TNF-a by triggering both caspase-induced apoptosis and ROS mediated mitochondrial dysfunction, the role of this pathway in hepatic cytotoxicity is not clear. An environment of oxidative stress would generate death ligands and subsequent production of caspase via activation of TNFR1; ceramide's role in apoptosis is a second messenger more down-stream in the TNF- $a$ related cytotoxic pathway. This mechanism could in part explain the triggering of apoptosis in steatohepatitis. Further, FFAs up-regulate NF- $\mathrm{KB}$ and induce transglutaminase 2 dependent apoptosis in hepatocytes and HepG2 cells mediated via ER stress [66]. These observations suggest ceramide induces apoptosis through different pathways and could play an important role in hepatocyte death in NASH.

\section{Linkage to human studies}

At this time human data linking ceramides to the pathogenesis of NAFLD or the progression from NAFLD to NASH are sparse. It has been shown that in obese subjects, ceramide and sphingomyelin concentrations are higher in the liver compared to subcutaneous and intraabdominal adipose tissue [67], and the presence of hepatic steatosis compared to absence of steatosis, in humans, correlates with increased adipose tissue ceramide concentrations [68]. It was also recently reported that lifestyle-induced weight loss resulted in a decrease in hepatic ceramide expression in obese patients with NAFLD, and a decrease in ceramide gene expression in the liver was associated with lower serum ceramides [69]. A significant reduction in plasma ceramide levels 6 months after bariatric surgery has also been reported [70]. This decrease correlated with improved insulin sensitivity. In addition, a novel link between plasma ceramides and diabetes might exist; obese patients with T2DM, compared to lean healthy controls, had increased plasma ceramides, and again this increase in ceramide correlated significantly with both the degree of insulin resistance and plasma TNFa [71]. It is quite possible that the mechanism for the higher plasma ceramides in obese individuals with insulin resistance is a direct result of an increased tissue flux of long chain FFAs leading to the stimulation of TNF-a mediated insulin resistance, a key pathogenic factor of NAFLD [72]. Since ceramides may increase TNF-a production through a positivefeedback loop, this would further worsen insulin resistance in these individuals. Using a new optimized and validated liquid chromatography electrospray ionization tandem mass spectrometry method, distinct ceramide species in different rat tissues (muscle, liver, and heart), and in human plasma were quantified, and it was shown that a supervised aerobic exercise intervention significantly reduces plasma ceramides in obese individuals with T2DM [73, 74]. Further, a decrease in the longer chain saturated C14:0 ceramide was also noted and was correlated with a greater improvement in insulin sensitivity. This suggests that specific ceramides, such as C14:0 may be important therapeutic targets for reversing insulin resistance, and this may also extend to the treatment of NAFLD. Further, there is a 
report that plasma ceramide levels are higher in hypertensive patients when compared to normotensive subjects [75]. Also, reductions in metabolic syndrome and cardiovascular risk factors including apolipoprotein-B100 and the ApoB100/ApoA1 ratio were correlated with plasma ceramides in obese patients, at 6 months after gastric bypass surgery [76]. In contrast, in one small study diacylglycerols, but not ceramides, were significantly increased in obese humans with NAFLD [77]. Of note, these patients had steatosis without significant inflammation, suggesting that the metabolic environment was less likely to lead to excessive production of ceramides. Collectively, these observations support a role for ceramide sphingolipids in obesity-induced T2DM, metabolic syndrome, as well as NAFLD in obese humans.

\section{Concluding remarks and future directions}

The involvement of ceramides in the pathogenesis of diabetes and the metabolic syndrome, including insulin resistance in the setting of obesity, has led to considerable interest in the role of sphingolipids in the development of NAFLD. Ceramides are important second messengers that interact with several pathways involved in insulin resistance; oxidative stress, inflammation, and apoptosis, all of which are linked to NAFLD (Table 1). Several risk factors for the progression of NAFLD such as inflammatory cytokines including TNF-a and IL-6, and decreased adiponectin along with oxidative stress are associated with ceramide production in hepatocytes. It is likely that the increase in inflammatory cytokines and oxidative stress that characterizes NASH is more conducive to the generation of ceramides. Ceramides could then further fuel the cellular damage caused by inflammation, worsening insulin resistance and promoting mitochondrial dysfunction and oxidative stress, thus facilitating the development of NASH. It appears that most of the cellular effects of ceramides are associated with the TNF- $a$ pathway, a key cytokine whose unopposed activity can promote steatosis and steatohepatitis. These observations lead us to suggest that the role of ceramides in NAFLD and its progression to NASH could be due in part to second messenger effects via TNF- $a$ mediated hepatic damage. Herein, we propose a mechanism by which ceramides contribute to the development and progression of NASH. This mechanism requires further investigation in models of steatohepatitis in human subjects (Fig. 2). If the role of ceramides as a contributing factor to NASH is established, these findings could have both diagnostic and therapeutic implications in NASH. While plasma ceramides could be used as important biomarkers in NASH, the availability of specific ceramide blocking agents could provide a potential therapeutic target in this patient population.

\section{Acknowledgments}

Funding: This research was supported in part by NIH grant RO1 DK089547 (JPK), and NIH National Center for Research Resources, CTSA 1UL1RR024989, Cleveland, Ohio. MP was supported by NIH grant T32 DK061917.

\section{References}

1. McCullough AJ. Pathophysiology of nonalcoholic steatohepatitis. J Clin Gastroenterol. 2006; 40(Suppl 1):S17-29. [PubMed: 16540762]

2. Choi SS, Diehl AM. Hepatic triglyceride synthesis and nonalcoholic fatty liver disease. Curr Opin Lipidol. 2008; 19:295-300. [PubMed: 18460922]

3. Hannun YA, Obeid LM. Principles of bioactive lipid signalling: lessons from sphingolipids. Nat Rev Mol Cell Biol. 2008; 9:139-150. [PubMed: 18216770]

4. Summers SA. Ceramides in insulin resistance and lipotoxicity. Prog Lipid Res. 2006; 45:42-72. [PubMed: 16445986]

5. Malaguarnera M, et al. Molecular mechanisms involved in NAFLD progression. J Mol Med (Berl). 2009; 87:679-695. [PubMed: 19352614] 
6. Gill JM, Sattar N. Ceramides: a new player in the inflammation-insulin resistance paradigm? Diabetologia. 2009; 52:2475-2477. [PubMed: 19802602]

7. Mari M, Fernandez-Checa JC. Sphingolipid signalling and liver diseases. Liver Int. 2007; 27:440450. [PubMed: 17403183]

8. Begriche K, et al. Mitochondrial dysfunction in NASH: causes, consequences and possible means to prevent it. Mitochondrion. 2006; 6:1-28. [PubMed: 16406828]

9. Malhi H, et al. Apoptosis and necrosis in the liver: a tale of two deaths? Hepatology. 2006; 43:S3144. [PubMed: 16447272]

10. Dowman JK, et al. Pathogenesis of non-alcoholic fatty liver disease. QJM. 2010; 103:71-83. [PubMed: 19914930]

11. Gault CR, et al. An overview of sphingolipid metabolism: from synthesis to breakdown. Adv Exp Med Biol. 2010; 688:1-23. [PubMed: 20919643]

12. Frangioudakis G, et al. Saturated- and n-6 polyunsaturated-fat diets each induce ceramide accumulation in mouse skeletal muscle: reversal and improvement of glucose tolerance by lipid metabolism inhibitors. Endocrinology. 2010; 151:4187-4196. [PubMed: 20660065]

13. Chang ZQ, et al. Endotoxin activates de novo sphingolipid biosynthesis via nuclear factor kappa Bmediated upregulation of Sptlc2. Prostaglandins Other Lipid Mediat. 2011; 94:44-52. [PubMed: 21167294]

14. Holland WL, et al. Lipid-induced insulin resistance mediated by the proinflammatory receptor TLR4 requires saturated fatty acid-induced ceramide biosynthesis in mice. J Clin Invest. 2011; 121:1858-1870. [PubMed: 21490391]

15. Clarke CJ, et al. The extended family of neutral sphingomyelinases. Biochemistry. 2006; 45:11247-11256. [PubMed: 16981685]

16. Nikolova-Karakashian M, et al. Role of neutral sphingomyelinases in aging and inflammation. Subcell Biochem. 2008; 49:469-486. [PubMed: 18751923]

17. Clarke CJ, et al. Neutral sphingomyelinase 2 (nSMase2) is the primary neutral sphingomyelinase isoform activated by tumour necrosis factor-alpha in MCF-7 cells. Biochem J. 2011; 435:381-390. [PubMed: 21303347]

18. Holland WL, Summers SA. Sphingolipids, insulin resistance, and metabolic disease: new insights from in vivo manipulation of sphingolipid metabolism. Endocr Rev. 2008; 29:381-402. [PubMed: 18451260]

19. Llacuna L, et al. Critical role of acidic sphingomyelinase in murine hepatic ischemia-reperfusion injury. Hepatology. 2006; 44:561-572. [PubMed: 16941686]

20. Novgorodov SA, et al. Novel pathway of ceramide production in mitochondria: thioesterase and neutral ceramidase produce ceramide from sphingosine and acyl-CoA. J Biol Chem. 2011; 286:25352-25362. [PubMed: 21613224]

21. Nikolova-Karakashian MN, Rozenova KA. Ceramide in stress response. Adv Exp Med Biol. 2010; 688:86-108. [PubMed: 20919648]

22. Ichi I, et al. Increase of ceramide in the liver and plasma after carbon tetrachloride intoxication in the rat. J Nutr Sci Vitaminol (Tokyo). 2007; 53:53-56. [PubMed: 17484380]

23. Lightle $S$, et al. Elevation of ceramide in serum lipoproteins during acute phase response in humans and mice: role of serine-palmitoyl transferase. Arch Biochem Biophys. 2003; 419:120128. [PubMed: 14592455]

24. Gentile CL, Pagliassotti MJ. The role of fatty acids in the development and progression of nonalcoholic fatty liver disease. J Nutr Biochem. 2008; 19:567-576. [PubMed: 18430557]

25. Malhi H, Gores GJ. Molecular mechanisms of lipotoxicity in nonalcoholic fatty liver disease. Semin Liver Dis. 2008; 28:360-369. [PubMed: 18956292]

26. Trauner M, et al. Fatty liver and lipotoxicity. Biochim Biophys Acta. 2010; 1801:299-310. [PubMed: 19857603]

27. Puri P, et al. A lipidomic analysis of nonalcoholic fatty liver disease. Hepatology. 2007; 46:10811090. [PubMed: 17654743] 
28. Wei Y, et al. Saturated fatty acids induce endoplasmic reticulum stress and apoptosis independently of ceramide in liver cells. Am J Physiol Endocrinol Metab. 2006; 291:E275-81. [PubMed: 16492686]

29. Chocian G, et al. High fat diet induces ceramide and sphingomyelin formation in rat's liver nuclei. Mol Cell Biochem. 2010; 340:125-131. [PubMed: 20174962]

30. Albi E, et al. Phosphatidylcholine/sphingomyelin metabolism crosstalk inside the nucleus. Biochem J. 2008; 410:381-389. [PubMed: 18001268]

31. Yetukuri L, et al. Bioinformatics strategies for lipidomics analysis: characterization of obesity related hepatic steatosis. BMC Syst Biol. 2007; 1:12. [PubMed: 17408502]

32. Yang G, et al. Central role of ceramide biosynthesis in body weight regulation, energy metabolism, and the metabolic syndrome. Am J Physiol Endocrinol Metab. 2009; 297:E211-24. [PubMed: 19435851]

33. Holland WL, et al. Inhibition of ceramide synthesis ameliorates glucocorticoid-, saturated-fat, and obesity-induced insulin resistance. Cell Metab. 2007; 5:167-179. [PubMed: 17339025]

34. Comi C. Fas-mediated T-cell apoptosis in chronic inflammatory demyelinating polyneuropathy. J Peripher Nerv Syst. 2011; 16(Suppl 1):45-47. [PubMed: 21696498]

35. Wueest $\mathrm{S}$, et al. Deletion of Fas in adipocytes relieves adipose tissue inflammation and hepatic manifestations of obesity in mice. J Clin Invest. 2010; 120:191-202. [PubMed: 19955656]

36. Pagadala M, et al. Predictors of steatohepatitis and advanced fibrosis in non-alcoholic fatty liver disease. Clin Liver Dis. 2009; 13:591-606. [PubMed: 19818307]

37. Semple RK, et al. Postreceptor insulin resistance contributes to human dyslipidemia and hepatic steatosis. J Clin Invest. 2009; 119:315-322. [PubMed: 19164855]

38. JeBailey L, et al. Ceramide- and oxidant-induced insulin resistance involve loss of insulindependent Rac-activation and actin remodeling in muscle cells. Diabetes. 2007; 56:394-403. [PubMed: 17259384]

39. Bikman BT, Summers SA. Ceramides as modulators of cellular and whole-body metabolism. J Clin Invest. 2011; 121:4222-4230. [PubMed: 22045572]

40. Ribaux PG, Iynedjian PB. Analysis of the role of protein kinase B (cAKT) in insulin-dependent induction of glucokinase and sterol regulatory element-binding protein 1 (SREBP1) mRNAs in hepatocytes. Biochem J. 2003; 376:697-705. [PubMed: 14505487]

41. Li DD, et al. The pivotal role of c-Jun NH2-terminal kinase-mediated Beclin 1 expression during anticancer agents-induced autophagy in cancer cells. Oncogene. 2009; 28:886-898. [PubMed: 19060920]

42. Schenk S, et al. Insulin sensitivity: modulation by nutrients and inflammation. J Clin Invest. 2008; 118:2992-3002. [PubMed: 18769626]

43. Schwabe RF, Brenner DA. Mechanisms of Liver Injury. I TNF-alpha-induced liver injury: role of IKK, JNK, and ROS pathways. Am J Physiol Gastrointest Liver Physiol. 2006; 290:G583-9. [PubMed: 16537970]

44. Wieckowska A, et al. Increased hepatic and circulating interleukin-6 levels in human nonalcoholic steatohepatitis. Am J Gastroenterol. 2008; 103:1372-1379. [PubMed: 18510618]

45. de Mello VD, et al. Link between plasma ceramides, inflammation and insulin resistance: association with serum IL-6 concentration in patients with coronary heart disease. Diabetologia. 2009; 52:2612-2615. [PubMed: 19669729]

46. Miller RA, et al. Adiponectin suppresses gluconeogenic gene expression in mouse hepatocytes independent of LKB1-AMPK signaling. J Clin Invest. 2011; 121:2518-2528. [PubMed: 21606593]

47. Polyzos SA, et al. Serum total adiponectin in nonalcoholic fatty liver disease: a systematic review and meta-analysis. Metabolism. 2011; 60:313-326. [PubMed: 21040935]

48. Buechler C, et al. Adiponectin, a key adipokine in obesity related liver diseases. World $\mathrm{J}$ Gastroenterol. 2011; 17:2801-2811. [PubMed: 21734787]

49. Holland WL, et al. Receptor-mediated activation of ceramidase activity initiates the pleiotropic actions of adiponectin. Nat Med. 2011; 17:55-63. [PubMed: 21186369] 
50. Kojima H, et al. Mitochondrial abnormality and oxidative stress in nonalcoholic steatohepatitis. Alcohol Clin Exp Res. 2007; 31:S61-6. [PubMed: 17331168]

51. Argo CK, Caldwell SH. Epidemiology and natural history of non-alcoholic steatohepatitis. Clin Liver Dis. 2009; 13:511-531. [PubMed: 19818302]

52. Mantena SK, et al. Mitochondrial dysfunction and oxidative stress in the pathogenesis of alcoholand obesity-induced fatty liver diseases. Free Radic Biol Med. 2008; 44:1259-1272. [PubMed: 18242193]

53. Colombini M. Ceramide channels and their role in mitochondria-mediated apoptosis. Biochim Biophys Acta. 2010; 1797:1239-1244. [PubMed: 20100454]

54. Mari M, et al. Mitochondrial free cholesterol loading sensitizes to TNF- and Fas-mediated steatohepatitis. Cell Metab. 2006; 4:185-198. [PubMed: 16950136]

55. Mari M, et al. Mechanism of mitochondrial glutathione-dependent hepatocellular susceptibility to TNF despite NF-kappaB activation. Gastroenterology. 2008; 134:1507-1520. [PubMed: 18343380]

56. Stiban J, et al. Ceramide synthases: roles in cell physiology and signaling. Adv Exp Med Biol. 2010; 688:60-71. [PubMed: 20919646]

57. Stiban J, et al. Ceramide synthesis in the endoplasmic reticulum can permeabilize mitochondria to proapoptotic proteins. J Lipid Res. 2008; 49:625-634. [PubMed: 18073406]

58. Brenner C, et al. GD3-7-aldehyde is an apoptosis inducer and interacts with adenine nucleotide translocase. Biochem Biophys Res Commun. 2010; 391:248-253. [PubMed: 19912988]

59. Malhi H, et al. Hepatocyte death: a clear and present danger. Physiol Rev. 2010; 90:1165-1194. [PubMed: 20664081]

60. Liu C, et al. Kupffer cells are associated with apoptosis, inflammation and fibrotic effects in hepatic fibrosis in rats. Lab Invest. 2010; 90:1805-1816. [PubMed: 20921949]

61. Guicciardi ME, Gores GJ. Apoptosis as a mechanism for liver disease progression. Semin Liver Dis. 2010; 30:402-410. [PubMed: 20960379]

62. Feldstein AE, et al. Cytokeratin-18 fragment levels as noninvasive biomarkers for nonalcoholic steatohepatitis: a multicenter validation study. Hepatology. 2009; 50:1072-1078. [PubMed: 19585618]

63. Bartke N, Hannun YA. Bioactive sphingolipids: metabolism and function. J Lipid Res. 2009; 50(Suppl):S91-6. [PubMed: 19017611]

64. Tamimi TI, et al. An apoptosis panel for nonalcoholic steatohepatitis diagnosis. J Hepatol. 2011; 54:1224-1229. [PubMed: 21145805]

65. Edelmann B, et al. Caspase- 8 and caspase-7 sequentially mediate proteolytic activation of acid sphingomyelinase in TNF-R1 receptosomes. EMBO J. 2011; 30:379-394. [PubMed: 21157428]

66. Kuo TF, et al. Free fatty acids induce transglutaminase 2-dependent apoptosis in hepatocytes via ER stress-stimulated PERK pathways. J Cell Physiol. 2012; 227:1130-1137. [PubMed: 21567402]

67. Kotronen A, et al. Comparison of lipid and fatty acid composition of the liver, subcutaneous and intra-abdominal adipose tissue, and serum. Obesity (Silver Spring). 2010; 18:937-944. [PubMed: 19798063]

68. Kolak M, et al. Adipose tissue inflammation and increased ceramide content characterize subjects with high liver fat content independent of obesity. Diabetes. 2007; 56:1960-1968. [PubMed: 17620421]

69. Promrat K, et al. Weight loss amelioration of non-alcoholic steatohepatitis linked to shifts in hepatic ceramide expression and serum ceramide levels. Hepatol Res. 2011; 41:754-762. [PubMed: 21794038]

70. Huang H, et al. Gastric Bypass Surgery Reduces Plasma Ceramide Subspecies and Improves Insulin Sensitivity in Severely Obese Patients. Obesity (Silver Spring). 2011

71. Haus JM, et al. Plasma ceramides are elevated in obese subjects with type 2 diabetes and correlate with the severity of insulin resistance. Diabetes. 2009; 58:337-343. [PubMed: 19008343]

72. Feldstein AE, et al. Free fatty acids promote hepatic lipotoxicity by stimulating TNF-alpha expression via a lysosomal pathway. Hepatology. 2004; 40:185-194. [PubMed: 15239102] 
73. Kasumov T, et al. Quantification of ceramide species in biological samples by liquid chromatography electrospray ionization tandem mass spectrometry. Anal Biochem. 2010; 401:154-161. [PubMed: 20178771]

74. Kasumov T, et al. Exercise Training Reduces Insulin Resistance and Plasma Ceramides in Obese Subjects, Including Type 2 Diabetics. Diabetes. 2009; 58:A244.

75. Spijkers LJ, et al. Hypertension is associated with marked alterations in sphingolipid biology: a potential role for ceramide. PLoS One. 2011; 6:e21817. [PubMed: 21818267]

76. Heneghan HM, et al. Reduced cardiovascular risk after bariatric surgery is linked to plasma ceramides, apolipoprotein-B100, and ApoB100/A1 ratio. Surg Obes Relat Dis. 2011 (Epub ahead of print).

77. Kotronen A, et al. Hepatic stearoyl-CoA desaturase (SCD)-1 activity and diacylglycerol but not ceramide concentrations are increased in the nonalcoholic human fatty liver. Diabetes. 2009; 58:203-208. [PubMed: 18952834]

78. Levy M, et al. nSMase 2 activation and trafficking are modulated by oxidative stress to induce apoptosis. Biochem Biophys Res Commun. 2006; 344:900-905. [PubMed: 16631623]

79. Li X, et al. Ceramide in redox signaling and cardiovascular diseases. Cell Physiol Biochem. 2010; 26:41-48. [PubMed: 20502003]

80. Huang WC, et al. Apoptotic sphingolipid ceramide in cancer therapy. J Lipids. 2011; 2011:565316. [PubMed: 21490804] 


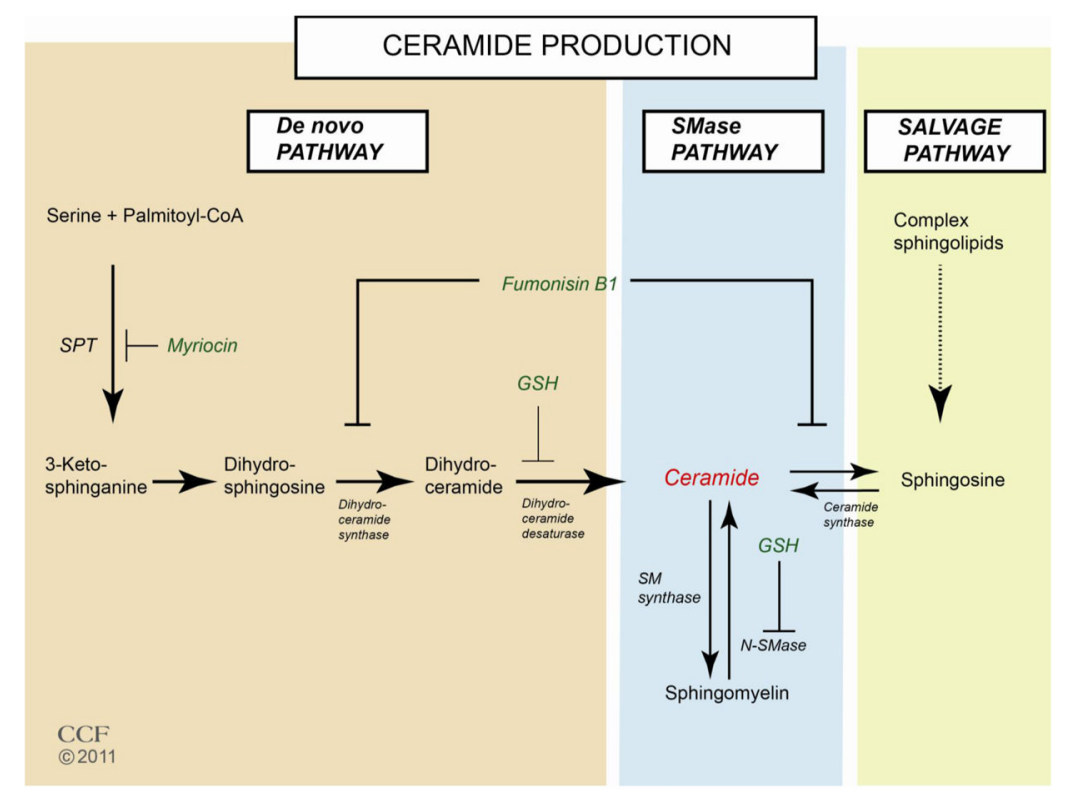

Figure 1. Ceramide biosynthesis through de novo, sphingomyolinase (SMase), and salvage pathways

Myriocin is a specific inhibitor of SPT, the enzyme that catalyzes the rate-limiting step in the de novo pathway. Fumanisin B1 inhibits (dehydro)ceramide synthase and down regulates both de novo and salvage pathways. The major cellular antioxidant glutathione is known to inhibit both (dehydro)ceramide synthase and N-SMase, and reduce ceramide production through de novo and SMase pathways. 


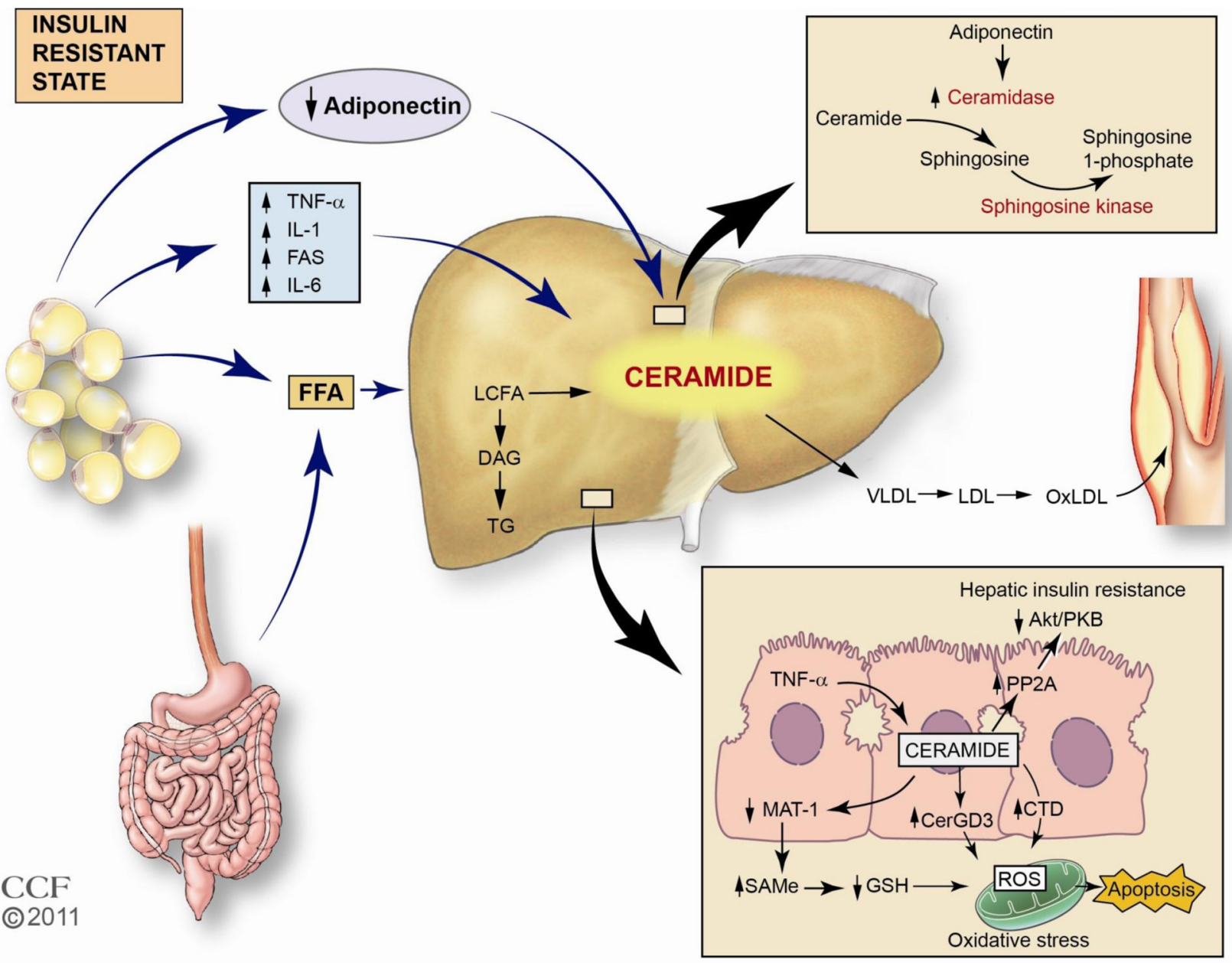

Figure 2. Potential role of ceramide in the development and progression of nonalcoholic fatty liver disease and related complications In this model we propose that obesity-induced insulin resistance gives rise to increased production and release of pro-inflammatory cytokines, including TNF-a, decreased adiponectin, and increased FFA release from an expanded adipose tissue mass. These mediators converge in the liver to facilitate ceramide production. Within the liver the increase in ceramides promotes hepatic insulin resistance through PP2A and attenuated Akt signaling, and apoptosis through a mitochondrial ROS mechanism. Adiponectin may also play an important role because a decrease in adiponectin would impair ceramide clearance through conversion to sphingosine 1-phosphate, thus facilitating greater ceramide accumulation within the tissue. The increase in hepatic ceramides likely contributes to the observed increase in plasma cermide levels in obese patients with T2DM, and may also play a role in skeletal muscle insulin resistance.

TNF-a, tumor necrosis factor; IL-6, Interleukin-6; 1L-1, Interleukin-1; FFA, free fatty acid; LCFA, long chain fatty acid, SphK, sphingosine kinase; DAG, diacylglycerol, TGL, triglyceride; MAT 1A, methionine adenosyltransferase 1A; SAMe, S-adenosylmethionine; GSH, glutathione; PP2A, protein phosphatase 2A; PKB, protein kinase B; Cer, ceramide; GD3, ganglioside GD3;CDase, ceramidase; SIP, sphingosine 1-phosphate; ROS, reactive oxygen species. 
Table 1

Mediators of ceramide production and resulting physiological effects that may contribute to the etiopathogenesis of steatosis and steatohepatitis

\begin{tabular}{|l|l|}
\hline Effectors of ceramide production & REF \\
\hline O Increased hepatic free fatty acid influx & {$[28,29,33]$} \\
OIncreased TNF-a & {$[7]$} \\
ODecreased adiponectin & {$[49]$} \\
OIncreased IL-1 & {$[18]$} \\
OProduction of ROS and oxidative stress & {$[78]$} \\
\hline Physiological effects of ceramides & REF \\
\hline OIncreased cytokine expression & {$[7,18]$} \\
OInsulin resistance & {$[32,33,40]$} \\
OMitochondrial dysfunction and decreased fat oxidation & {$[18,53]$} \\
OOxidative stress & {$[79]$} \\
OTriggering of cellular apoptosis & {$[53,80]$} \\
ODown-regulation of methionine adenosyltransferase 1A expression and decrease in the redox ratio & {$[7]$} \\
OIncreased lipoprotein aggregation & {$[18,79]$} \\
\hline
\end{tabular}

